\title{
Sciendo
}

\section{BONE HOMEOSTASIS IN EXPERIMENTAL FUMONISINS INTOXICATION OF RATS*}

\author{
Halyna Rudyk ${ }^{1 \bullet}$, Ewa Tomaszewskaa ${ }^{2 \star}$, Ihor Kotsyumbas ${ }^{1}$, Siemowit Muszyński ${ }^{3}$, \\ Agnieszka Tomczyk-Warunek ${ }^{2,3}$, Sylwia Szymańczyk ${ }^{2}$, Piotr Dobrowolski ${ }^{4}$, Dariusz Wiącek ${ }^{5}$, \\ Daniel Kamiński ${ }^{6}$, Oksana Brezvyn ${ }^{1}$
}
${ }^{1}$ The State Scientific Research Control Institute of Veterinary Medicinal Products and Feed Additives (SCIVP), Donetska 11, 79019 Lviv, Ukraine
${ }^{2}$ Department of Animal Physiology, University of Life Sciences in Lublin, Akademicka 12, 20-950 Lublin, Poland
${ }^{3}$ Department of Physics, University of Life Sciences, Akademicka 13, 20-950 Lublin, Poland ${ }^{4}$ Department of Comparative Anatomy and Anthropology, Maria Curie-Skłodowska University, Akademicka 19, 20-033 Lublin, Poland
${ }^{5}$ Institute of Agrophysics, Polish Academy of Sciences, Doświadczalna 4, 20-001 Lublin, Poland ${ }^{6}$ Department of Crystallography, Maria Curie-Skłodowska University, Plac Marii Curie-Skłodowskiej 3, 20-031 Lublin, Poland
•Corresponding authors: ewaRST@interia.pl; galusik.77@gmail.com

\begin{abstract}
Fumonisins are strongly toxic metabolites of Fusarium proliferatum and Fusarium verticillioides commonly present in corn-based feed. The aim of the study was to evaluate bone homeostasis in experimental fumonisins B1 and B2 intoxication of rats, a vertebrate animal model of toxicological studies, as still little is known about the possible disturbing effect of fumonisins on bone homeostasis. Adolescent (5-week-old) male Wistar rats were randomly assigned into a control group and a group FB intoxicated with fumonisins by daily intragastric administration of fumonisins at the dose of $90 \mathrm{mg} / \mathrm{kg}$ of body weight per animal in the FB group for 21 days. The fumonisin intoxication did not affect body and bone mass, although the mechanical and geometric properties were decreased in fumonisin-intoxicated rats. Bone volumetric and mineral density did not differ between groups, but bone mineral content and bone ash percentage was lower in the FB group. Detailed analysis showed that $\mathrm{Ca}, \mathrm{Cu}, \mathrm{Fe}, \mathrm{Mn}, \mathrm{Sr}$, and $\mathrm{Zn}$ bone content significantly decreased in fumonisin intoxicated rats and the alterations in structure of bone mineral phase (reduction of the apatite-bone crystals size) were noted. While the negative structural alterations in growth plate and articular cartilages were also observed, fumonisin intoxication improved histomorphometrical parameters of trabecular bone. Concluding, the dose of fumonisins used in the present study caused hepatotoxic effect, which was sufficient to trigger the disturbance in mineral homeostasis resulting in altered bone metabolism and decreased mechanical endurance.
\end{abstract}

Key words: fumonisins, bones, rats, mechanical properties, histomorphometry

*Study funded by The State Scientific Research Control Institute of Veterinary Medical Product and Feed Additives and Statutory Research of Department of Physiology, University of Life Sciences in Lublin, no. WKF/DS-1. 
Fumonisins are strongly toxic metabolites of Fusarium proliferatum and Fusarium verticillioides that have been found not only in cereal grains (including maize) or crop products, but also in pelleted feed, what could indicate that fumonisins are resistant to the pelleting process. Among a number of different fumonisins B (FB) mycotoxins only B1 and B2 are toxicologically significant because of their relative abundance (Šegvić and Pepeljnjak, 2001). A worldwide survey showed that FB were found in over $50 \%$ of investigated feed and feed raw material samples and the contamination level in cereals varied from 0.02 to $25.9 \mathrm{mg} / \mathrm{kg}$, and from 0.05 to $11.3 \mathrm{mg} /$ $\mathrm{kg}$, respectively (Placinta et al., 1999; Streit et al., 2015).

Although FB2 differs structurally from FB1 in the number and position of hydroxyl groups, both have been shown to be inhibitors of de novo sphingolipid synthesis. For this reason FBs are involved in the regulation of cell growth, differentiation, and even cancer transformation (Ross et al., 1992). The most susceptible to the effects of FBs are horses and pigs, in which clinical problems have been reported (equine leukoencephalomalacia; porcine pulmonary oedema) (EFSA, 2018). Also, hepatic or kidney toxicities are observed in equines, pigs, sheep, rodents and poultry (Riley and Voss, 2006; Antonissen et al., 2015). On the other hand, poultry are considered to be quite resistant toward the deleterious effects of FBs. Bioavailability and toxicity of FBs is poor in ruminants compared to monogastric animals. It means that FBs toxic dose or clinical signs of toxicity vary between different species and depend on administration routes as well as sex or age (Ledoux et al., 1992).

Bone formation and growth is controlled by a complex group of feedback processes that depend on several biological and environmental factors like exposure to toxicants (Álvarez-Lloret et al., 2009; Hodgson et al., 2008; Rodríguez-Estival et al., 2013). Our earlier toxicological studies have demonstrated that bone tissue is highly sensitive to many types of toxic substances like heavy metals (cadmium, lead) or mycotoxins (deoxynivalenol), which affect bone composition, mineralization and mechanical strength (Tomaszewska et al., 2016, 2017 a, 2018 a).

Little is known about the possible disturbing effects of FBs on bone homeostasis and development in the vertebrate/mammals, as the only studies were performed over 20 years ago on pregnant rats (Lebepe-Mazur et al., 1995) and chickens (Wu et al., 1995). However, it is believed that FBs are possible etiological factors in skeletal problems and diets containing FBs culture material increased the incidence of leg deformity in livestock animals (Kierończyk et al., 2017; Lee et al., 2018).

Accordingly, this study was designed to evaluate bone homeostasis in experimental fumonisin intoxication of rats, a vertebrate animal model of toxicological studies. The analyzed traits included measurements of bone densitometry and osteometric parameters, analysis of microelements content, comprehensive analyses of the mechanical characteristics of bone midshaft and microstructure of the articular and growth plate cartilages, assessment of crystallinity of mineral phase and collagen structure (distribution) in bone tissues. Applied analytical techniques allowed providing detailed information about the material composition of bone and structural organization of both inorganic and organic bone components. The information acquired in the presented study may expand the knowledge about the influence of fumonisins on bone structure/homeostasis. 


\section{Material and methods}

All experimental procedures were approved by The Local Ethics Committee in the State Scientific Research Control Institute of Veterinary Medicinal Products and Feed Additives (SCIVP), Lviv, Ukraine, and complied with the Directive 2010/63/ EU of the European Parliament and of the Council on the protection of animals used for scientific purposes.

\section{Preparation of fumonisins extract}

FBs culture material was produced in vitro from a culture of $F$. verticillioides (Laboratory of Mycotoxicology, Institute of Veterinary Medicine of The National Academy of Agrarian Sciences of Ukraine, Kiev, Ukraine) in tryptone glucose yeast (TGY) broth medium in Petri dishes. Autoclave sterilized corn grains were plated equidistantly in Petri dishes and incubated at $25^{\circ} \mathrm{C}$ for $4-5$ weeks. Next, the contaminated grains were ground and extracted with ethanol for one day. The obtained extract was filtered and concentrated by evaporation. In experiments, the extract was given in distilled water. As shown by HPLC analysis (National Veterinary Research Institute, Puławy, Poland), the contaminated corn contained $75 \%$ of FB1 and $25 \%$ of FB2 (182.0 and $59.5 \mu \mathrm{g} / \mathrm{kg}$, respectively).

\section{Animals, experimental procedures and tissue collection}

The study was conducted in vivarium conditions of the State Scientific-Research Control Institute of Veterinary Medicinal Products and Feed Additives in Lviv, Ukraine. In the main experiment, adolescent (5-week-old) male Wistar rats $(n=40)$ after individual weighing were placed separately in polypropylene cages $(380 \times 200$ $\times 590 \mathrm{~mm}$ ) and left for one week to acclimate to new laboratory conditions. The rats were held at a temperature of $21 \pm 3^{\circ} \mathrm{C}$, humidity of $55 \pm 5 \%$, and in $12 \mathrm{~h} / 12 \mathrm{~h}$ day/ night cycle; had a free access to water. After the acclimatization period, the animals were randomly distributed into a control group ( $C$ group; $n=20)$ not intoxicated with fumonisins and a group intoxicated with fumonisins (FB group; $n=20$ ). The rats in both groups were fed ad libitum standard laboratory rodents diet, formulated to meet nutritional requirements specified in AIN-93M directive (Reeves et al., 1993). Clinically detectable fumonisin toxicosis (based on liver tests) was induced by intragastric daily administration of fumonisins (FB1+FB2) at the dose of $90 \mathrm{mg} / \mathrm{kg}$ of body weight per animal in the FB group for 21 days. As the median lethal dose $\left(\mathrm{LD}_{50}\right)$ value of FB for rats is not known (McKean et al., 2006), in the preliminary study $\mathrm{LD}_{50}(900 \mathrm{mg} / \mathrm{kg}$ body weight) and dose triggering clinical signs were established. Based on these earlier results, administrated dose of FB1+FB2 (75\% and 25\%, respectively), equal to 0.1 of established $\mathrm{LD}_{50}$ value, was sufficient to receive clinical signs in all of the infected animals in this study. On the 21st day of the experiment rats were fasted for 24 hours and weighed. In each group, 6 animals with the weight closest to the group average were euthanized by $\mathrm{CO}_{2}$ inhalation.

Blood was immediately sampled directly from the heart of rats into $6 \mathrm{ml}$ vacuum tubes, and serum was immediately separated by centrifugation and frozen at $-80^{\circ} \mathrm{C}$ for further analysis. 
Both femora were dissected, and after the individual measurements of bone mass and length they were wrapped in gauze soaked in isotonic $\mathrm{PhS}$ and frozen at $-25^{\circ} \mathrm{C}$ for further analysis. In subsequent stages of analyses, the bones collected from the left side of rats were used for osteometric measurements and histomorphometrical analysis, while right femora were subjected to densitometry, strength tests, and were ashed for further analysis (ash content, diffractometry, mineral composition).

\section{Blood serum analyses}

Total calcium and phosphorus concentrations were determined with the colorimetric method (Tomaszewska et al., 2016).

\section{Bone densitometry and mechanical testing}

Bone mineral density and bone mineral content were determined using the dual-energy X-ray absorptiometry method on XR 43 densitometer (Norland, Fort Atkinson, WI, USA) (Tomaszewska et al., 2017 b). The mechanical (structural and material) properties of bones were assessed on the basis of the 3-point bending test performed on Zwick Z010 universal testing machine (Zwick-Roell GmbH \& Company KG, Ulm, Germany). The load was applied with a constant speed of $10 \mathrm{~mm} /$ min until fracture. The values of the yield load and ultimate load were determined and from the registered relationship between the applied force and bone deflection bone structural properties (elastic energy, work to fracture, stiffness) were calculated (Muszyński et al., 2017) using Origin 9.0 software (OriginLab Co., Northampton, MA, USA).

\section{Osteometric measurements and determination of bone material properties}

Femur mid-diaphysis cross-sectional geometry was determined on the basis of osteometric measurements. They included determination of both external and internal diameters of the mid-diaphysis cross-section (both in medial-lateral and cranial-caudal plane) with the electronic caliper. The calculated geometric properties were: cortical cross-sectional area, mean relative wall thickness, and cortical index (Muszyński et al., 2017). Moreover, as during the strength analysis the bone was loaded in the cranial-caudal plane, vertical cortical index, cross-sectional moment of inertia and radius of gyration about medial-lateral axis were calculated (Muszyński et al., 2017).

Whole-bone material properties were calculated on the basis of determined structural properties and geometry of the bone mid-diaphysis using standard beam-theory equations (Muszyński et al., 2017). The ascertained material properties included: Young modulus of elasticity, yield strain, ultimate strain, bending moment, yield stress, and ultimate stress.

\section{Histomorphometric analysis}

Bone sections for histomorphometry were obtained from fixed with formalin, decalcified in EDTA and embedded in paraffin distal bone samples. From each individual, 4- $\mu \mathrm{m}$ thick sagittal sections containing cartilage and bone were cut with a microtome from the middle part of the lateral condyle. Three slides of four sections 
for each specimen were made. Sections were stained with trichrome (Goldner's) method to assess basal morphology of the articular and growth plate cartilages, and Safranine O staining to evaluate proteoglycans content in articular cartilage (Suvara et al., 2013). The Picrosirius red (PSR) staining was used to assess the morphology of trabecular bone and cancellous bone by the evaluation of the distribution of thin (immature) and thick (mature) collagen fibres (Rich and Wihittaker, 2005). Maturity of collagen was estimated by calculating, as pixel count, the percentage of green, immature collagen fibres in PSR sections, as seen in polarized light (Blicharski et al., 2017). The growth plate total thickness, as well as the thickness of reserve zone (I), the proliferative zone (II), the hypertrophic zone (III) and the ossification (IV) of cartilage were estimated (Tomaszewska et al., 2017 c). Similarly, in articular cartilage the thicknesses of the horizontal (I), transitional (II) and radial (III) zones were measured (Tomaszewska et al., 2013). All analyses of microscopic images were performed using CellSens software (Olympus, Tokyo, Japan). For trabecular region in bone metaphysis and epiphysis common microarchitectural descriptors were calculated using the public domain ImageJ software (Schneider et al., 2012). They included bone volume to tissue volume ratio (BV/TV), trabecular thickness (Tb.Th), trabecular separation (Tb.Sp) and trabecular number (Tb.N). A detailed description of the parameters used can be found elsewhere (Tomaszewska et al., 2018 a).

All chemicals and solvents were at least of analytical reagent grade and were obtained from Sigma-Aldrich (St. Louis, MO, USA), Thermo Fisher Scientific (Waltham, MA, USA) or Avantor Performance Materials Poland S.A. (Gliwice, Poland).

\section{Bone volumetric density, ash content and mineral composition}

The measurement of bone tissue density (volumetric density) was performed with a helium gas pycnometer as described previously (Tomaszewska et al., 2015; $2018 \mathrm{~b}$ ). Prior to the analysis, the samples were dried in an oven at $105^{\circ} \mathrm{C}$ for $24 \mathrm{~h}$. After the measurement, samples were ashed in a muffle furnace at $500^{\circ} \mathrm{C}$ for $24 \mathrm{~h}$. Bone ash percentage was expressed relative to the bone dry mass.

The mineral composition of bone was determined using ICP-OES spectrometry (iCAP Series 6500, Thermo Scientific, Waltham, MA, USA) in ashed bone samples. The TraceCERT multi-element stock solution (Sigma-Aldrich) was used to prepare reference standards. The macro- and microelements content was expressed as in crude ash.

\section{Structural analysis of bone mineral phase}

The crystallinity of bone mineral phase was measured in ashed bone samples pulverized into a finely divided white powder by X-ray diffraction (XRD) method performed with an Empyrean powder diffractometer (PANalytical, Almelo, The Netherlands) (Tomaszewska et al., 2017). The mean size of the nanocrystallites was calculated according to the Scherrer equation (Schreiner and Jenkins, 1983) with the shape constant of 0.9 and apparatus broadening of $0.01 \mathrm{deg}$. The peaks corresponding to Miller indices (200), (3-10) and (300) were taken for the calculation of apatite-bone crystals size in a-b plane, while the size in the $\mathrm{z}$ axis was calculated on the basis of indices (002) and (004) (Rajesh et al., 2012). The peak position and FWHM 
(full width at half maximum) were calculated from the fits of the Voight function to each peak with use of the Origin software.

\section{Statistical analysis}

An individual animal was considered as the experimental unit. The data were analyzed using Statistica 13 software (TIBCO Software Inc., Palo Alto, CA, USA). The normality of data distribution was tested using the Shapiro-Wilk test. A comparison between normally distributed data was carried out using Student's t test. When the data did not meet the assumptions for parametric tests, the Mann-Whitney U test was applied. For all tests a P-value less than 0.05 was considered statistically significant. All results are expressed as means $\pm \mathrm{SD}$ (standard deviation).

\section{Results}

The initial mean body mass of the rats did not differ among the groups. The control and FB-intoxicated rats weighed $211.9 \pm 2.7 \mathrm{~g}$ and $223.1 \pm 3.7 \mathrm{~g}$, respectively. The control group showed a very slight, but not significant increase in body mass at the end of experiment and reached the value of $235.0 \pm 1.7 \mathrm{~g}$, while FB-intoxicated rats weighed about $219.1 \pm 2.7 \mathrm{~g}$.

\section{Results of bone analyses}

The FB treatment did not influence the mass of the femur, although reduced its length (Table 1). The values of bone mineral density and bone tissue density did not differ between groups, but bone mineral content and bone ash percentage were decreased in FB-intoxicated rats. However, the values of both internal diameters increased after FB intoxication (Table 2). As a result, mean relative wall thickness, vertical cortical index decreased. Among structural properties of femur mid-diaphyseal part yield load, elastic energy, and work to fracture were significantly decreased in FB-intoxicated rats (Table 3). Among all of determined bone material parameters only ultimate strain significantly increased in FB group while the values of the remaining parameters, except yield strain which did not differ between groups, were reduced in that group (Table 4).

Table 1. Basal morphometry, densitometry, and ash of femur obtained from adolescent control and FB-intoxicated rats

\begin{tabular}{l|c|c|c}
\hline \multirow{2}{*}{\multicolumn{1}{c|}{ Densitometry properties }} & \multicolumn{2}{c}{ Group } & \multirow{2}{*}{ P-value } \\
\cline { 2 - 3 } & control & fumonisin & \\
\hline Bone mass $(\mathrm{g})$ & $0.548 \pm 0.047$ & $0.567 \pm 0.024$ & n.s. \\
Bone length $(\mathrm{mm})$ & $32.5 \pm 1.2$ & $30.6 \pm 0.6$ & $* *$ \\
Bone mineral density $\left(\mathrm{g} / \mathrm{cm}^{2}\right)$ & $0.222 \pm 0.020$ & $0.226 \pm 0.023$ & n.s. \\
Bone mineral content $(\mathrm{g})$ & $0.461 \pm 0.043$ & $0.388 \pm 0.045$ & $* *$ \\
Bone tissue density $\left(\mathrm{g} / \mathrm{cm}^{3}\right)$ & $2.40 \pm 0.16$ & $2.50 \pm 0.11$ & n.s. \\
Ash $(\%)$ & $50.14 \pm 1.1$ & $46.9 \pm 0.5$ & $* * *$ \\
\hline
\end{tabular}

Statistical significance: $\mathrm{n} . \mathrm{s} .-$ not significant $\mathrm{P}>0.05 ; * * \mathrm{P}<0.01 ; * * * \mathrm{P}<0.001$. 
Table 2. Geometric properties of mid-diaphyseal part of femora obtained from adolescent control and FB-intoxicated rats

\begin{tabular}{l|c|c|c}
\hline \multirow{2}{*}{\multicolumn{1}{c}{ Geometric properties }} & \multicolumn{2}{c}{ Group } & \multirow{2}{*}{ P-value } \\
\cline { 2 - 3 } & control & fumonisin & \\
\hline C-C plane external diameter $(\mathrm{mm})$ & $2.54 \pm 0.08$ & $2.61 \pm 0.09$ & n.s. \\
C-C plane internal diameter (mm) & $1.35 \pm 0.11$ & $1.59 \pm 0.06$ & $* * *$ \\
M-L plane external diameter (mm) & $3.35 \pm 0.21$ & $3.49 \pm 0.21$ & n.s. \\
M-L plane internal diameter $(\mathrm{mm})$ & $1.83 \pm 0.09$ & $1.96 \pm 0.07$ & $*$ \\
Cross-sectional area (mm $\left.{ }^{2}\right)$ & $4.71 \pm 0.23$ & $4.69 \pm 0.30$ & n.s. \\
Mean relative wall thickness & $0.92 \pm 0.11$ & $0.76 \pm 0.09$ & $*$ \\
Cortical index (\%) & $46.3 \pm 7.5$ & $41.6 \pm 6.9$ & n.s. \\
Vertical cortical index (\%) & $46.9 \pm 6.2$ & $39.1 \pm 5.6$ & $*$ \\
Cross-sectional moment of inertia $\left(\mathrm{mm}^{4}\right)$ & $2.46 \pm 0.62$ & $2.63 \pm 0.23$ & n.s \\
Radius of gyration (mm) & $0.72 \pm 0.06$ & $0.75 \pm 0.04$ & n.s. \\
\hline
\end{tabular}

Statistical significance: $\mathrm{n} . \mathrm{s} .-$ not significant $\mathrm{P}>0.05 ; * \mathrm{P}<0.05 ; * * * \mathrm{P}<0.001$.

C-C plane - cranial-caudal (vertical) plane; M-L plane - medial-lateral (horizontal) plane.

Table 3. Structural properties of mid-diaphyseal part of femora obtained from adolescent control and FB-intoxicated rats

\begin{tabular}{l|c|c|c}
\hline \multirow{2}{*}{\multicolumn{1}{c}{ Structural properties }} & \multicolumn{2}{c}{ Group } & \multirow{2}{*}{ P-value } \\
\cline { 2 - 3 } & control & fumonisin & \\
\hline Yield load (N) & $73 \pm 10$ & $51 \pm 6$ & $* * *$ \\
Ultimate load (N) & $112 \pm 9$ & $100 \pm 15$ & n.s. \\
Elastic energy (mJ) & $9.09 \pm 2.91$ & $5.43 \pm 0.94$ & $*$ \\
Work to fracture (mJ) & $47.6 \pm 5.3$ & $37.2 \pm 8.0$ & $*$ \\
Stiffness (N/mm) & $284 \pm 55$ & $255 \pm 44$ & n.s. \\
\hline
\end{tabular}

Statistical significance: $\mathrm{n} . \mathrm{s} .-$ not significant $\mathrm{P}>0.05 ; * \mathrm{P}<0.05 ; * * * \mathrm{P}<0.001$.

Table 4. Material properties of femur obtained from adolescent control and FB-intoxicated rats

\begin{tabular}{l|c|c|c}
\hline \multirow{2}{*}{\multicolumn{1}{c}{ Material properties }} & \multicolumn{2}{c|}{ Group } & \multirow{2}{*}{ P-value } \\
\cline { 2 - 3 } & control & fumonisin & \\
\hline Young modulus of elasticity $(\mathrm{GPa})$ & $4.92 \pm 1.02$ & $3.39 \pm 0.67$ & $*$ \\
Yield strain (\%) & $2.34 \pm 0.37$ & $2.19 \pm 0.34$ & n.s. \\
Ultimate strain (\%) & $5.44 \pm 0.94$ & $6.66 \pm 0.96$ & $*$ \\
Bending moment $(\mathrm{N} \times \mathrm{m})$ & $0.22 \pm 0.06$ & $0.15 \pm 0.02$ & $*$ \\
Yield stress $(\mathrm{MPa})$ & $115 \pm 32$ & $74 \pm 13$ & $*$ \\
Ultimate stress $(\mathrm{MPa})$ & $186 \pm 26$ & $158 \pm 11$ & $*$ \\
\hline
\end{tabular}

Statistical significance: $n . s .-$ not significant $\mathrm{P}>0.05 ; * \mathrm{P}<0.05$.

The intoxication with FB reduced the total thickness of articular cartilage and all of its zones (Table 5). The staining with Safranine O showed a difference in the content of proteoglycans (color intensity) in articular cartilage in control rats 
and FB-intoxicated ones (Figure 1). Lower proteoglycan content (very weak pink staining) was observed in the articular cartilage in FB-intoxicated rats, while more intense pink staining, however not strong, was in the control (Figure 1). Further, FBintoxicated rats had also reduced the total thickness of growth plate, which resulted from the lessening of all its zones except zone III, which was unaffected (Table 5).

Table 5. The morphology of growth plate cartilage and articular cartilage of femur obtained from adolescent control and FB-intoxicated rats

\begin{tabular}{l|c|c|c}
\hline \multirow{2}{*}{ Zone thickness } & \multicolumn{2}{c}{ Group } & \multirow{2}{*}{ P-value } \\
\cline { 2 - 3 } & control & fumonisin & \\
\cline { 2 - 3 } Growth plate cartilage & & & $* * *$ \\
Zone I (mm) & $46.3 \pm 12.0$ & $27.8 \pm 8.9$ & $* * *$ \\
Zone II (mm) & $92.4 \pm 13.5$ & $47.4 \pm 17.5$ & n.s. \\
Zone III (mm) & $42.5 \pm 6.7$ & $41.8 \pm 14.5$ & $* * *$ \\
Zone IV (mm) & $90.0 \pm 13.5$ & $65.4 \pm 14.4$ & $* * *$ \\
Total thickness (mm) & $297.7 \pm 32.5$ & $199.6 \pm 46.7$ & $* * *$ \\
Articular cartilage & & & $* * *$ \\
Zone I (mm) & $19.1 \pm 3.3$ & $16.1 \pm 2.8$ & $* * *$ \\
Zone II (mm) & $63.7 \pm 12.6$ & $33.0 \pm 8.6$ & $* * *$ \\
Zone III (mm) & $72.6 \pm 14.1$ & $54.1 \pm 20.6$ & \\
Total thickness (mm) & $206.1 \pm 40.7$ & $102.3 \pm 17.4$ & \\
\hline
\end{tabular}

Statistical significance: n.s. - not significant $\mathrm{P}>0.05 ; * * * \mathrm{P}<0.001$.

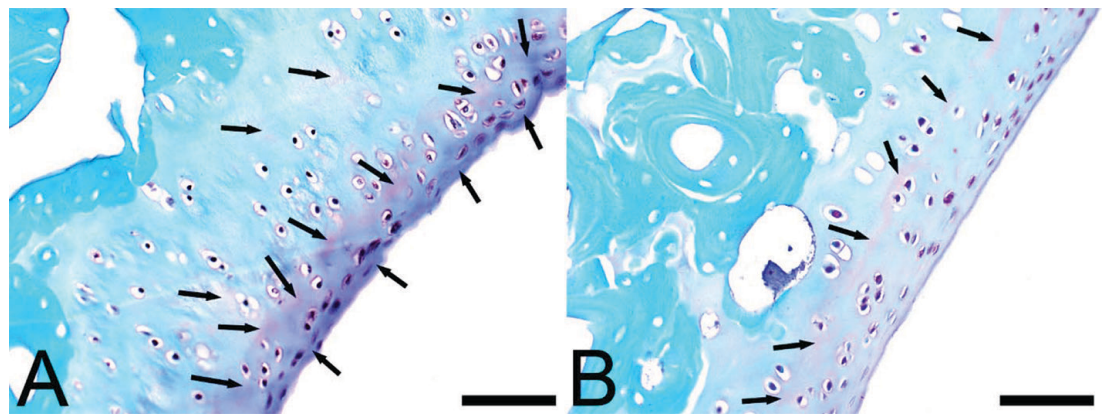

A - the control group, B - the FB group; scale bar - $50 \mu \mathrm{m}$.

Proteoglycan staining with SO (black arrows) showed lower proteoglycan content (displaying weaker staining) in the cartilage from the FB group, while rats from the control group demonstrated moderate staining linked with higher content of proteoglycans.

Figure 1. Representative images of safranin O (SO) staining carried out on formaldehyde-fixed sections of articular cartilage from the femur of adolescent rats FB-intoxicated for 21 days

Neither the BV/TV in epiphysis of femur nor Tb.Sp max in metaphysis were affected by FB intoxication (Table 6). All other determined histomorphometrical parameters were increased in FB-intoxicated rats, except trabecular number, which was significantly reduced in both epiphysis and metaphysis (Table 6; Figure 2). 
Table 6. Trabecular bone morphology of distal part of femur obtained from adolescent control and FBintoxicated rats

\begin{tabular}{|c|c|c|c|}
\hline \multirow{2}{*}{ Histomorphometry } & \multicolumn{2}{|c|}{ Group } & \multirow{2}{*}{ P-value } \\
\hline & control & fumonisin & \\
\hline \multicolumn{4}{|l|}{ Epiphysis } \\
\hline BV/TV (\%) & $50.2 \pm 4.0$ & $49.0 \pm 4.5$ & n.s. \\
\hline Tb.Th mean $(\mu \mathrm{m})$ & $34.5 \pm 5.1$ & $51.4 \pm 13.2$ & $* *$ \\
\hline Tb.Th max $(\mu \mathrm{m})$ & $87.4 \pm 14.6$ & $123.5 \pm 31.5$ & $* *$ \\
\hline Tb.Sp mean $(\mu \mathrm{m})$ & $56.0 \pm 5.1$ & $83.9 \pm 15.6$ & $* * *$ \\
\hline Tb.Sp max $(\mu \mathrm{m})$ & $129.5 \pm 25.4$ & $196.8 \pm 36.8$ & $* * *$ \\
\hline Tb.N $(\mu \mathrm{m})$ & $14.7 \pm 1.4$ & $9.9 \pm 1.7$ & $* * *$ \\
\hline \multicolumn{4}{|l|}{ Metaphysis } \\
\hline BV/TV (\%) & $44.8 \pm 0.9$ & $47.8 \pm 0.9$ & $* * *$ \\
\hline Tb.Th mean $(\mu \mathrm{m})$ & $39.3 \pm 2.7$ & $43.2 \pm 2.8$ & $*$ \\
\hline Tb.Th max $(\mu \mathrm{m})$ & $97.7 \pm 3.7$ & $106.1 \pm 1.8$ & $* * *$ \\
\hline Tb.Sp mean $(\mu \mathrm{m})$ & $74.9 \pm 1.8$ & $67.7 \pm 1.4$ & $* * *$ \\
\hline Tb.Sp max $(\mu \mathrm{m})$ & $173.8 \pm 22.3$ & $170.5 \pm 19.3$ & n.s \\
\hline Tb.N $(\mu \mathrm{m})$ & $12.1 \pm 0.8$ & $10.7 \pm 0.6$ & $* *$ \\
\hline
\end{tabular}

Statistical significance: $\mathrm{n} . \mathrm{s} .-$ not significant $\mathrm{P}>0.05 ; * \mathrm{P}<0.05 ; * * \mathrm{P}<0.01 ; * * * \mathrm{P}<0.001$.

$\mathrm{BV} / \mathrm{TV}$ - ratio of bone volume/tissue volume; Tb.Th - trabecular thickness; $\mathrm{Tb} . \mathrm{Sp}$ - trabecular separation; Tb.N - trabecular number.
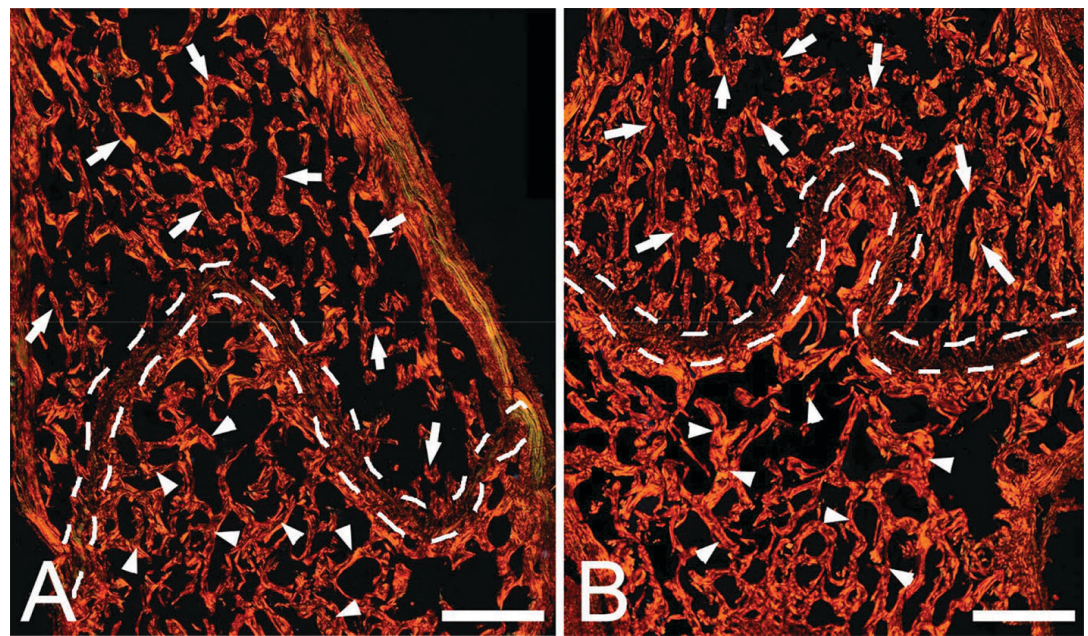

A - the control, group B - the FB group; scale bar - $100 \mu \mathrm{m}$.

In the center of each image a growth plate cartilage can be seen (white dotted line). Trabeculae of epiphysis are located above (white arrows) while trabeculae of metaphysis are located below growth plate cartilage (white arrowheads). Neither the BV/TV in epiphysis nor trabecular space in metaphysis were affected by FB intoxication. All other determined histomorphometrical parameters were increased in FB-intoxicated rats, except trabecular number, which was significantly reduced both in epiphysis and metaphysis.

Figure 2. Representative images of the Picrosirius red (PSR) staining carried out on formaldehyde-fixed sections from the femur (sections containing trabecular bone in metaphysis and epiphysis) of adolescent rats FB-intoxicated for 21 days 
The content of immature thin collagen in trabecular bone irrespective of the place of the determination was not changed in FB-intoxicated rats (Table 7). However, the content of immature collagen decreased in cancellous bone and articular cartilage (Table 7; Figure 3).

Table 7. Thin (immature) collagen content in femur obtained from adolescent control and FB-intoxicated rats

\begin{tabular}{l|c|c|c}
\hline \multirow{2}{*}{\multicolumn{1}{c}{ Thin collagen content }} & \multicolumn{2}{c}{ Group } & \multirow{2}{*}{ P-value } \\
\cline { 2 - 3 } & control & fumonisin & \\
\hline Trabecular bone, epiphysis (\%) & $0.61 \pm 0.32$ & $0.44 \pm 0.18$ & n.s. \\
Trabecular bone, metaphysis (\%) & $0.76 \pm 0.42$ & $0.65 \pm 0.33$ & n.s. \\
Cancellous bone (\%) & $4.15 \pm 1.30$ & $1.11 \pm 0.51$ & $* * *$ \\
Articular cartilage (\%) & $1.01 \pm 0.47$ & $0.12 \pm 0.07$ & $* * *$ \\
\hline
\end{tabular}

Statistical significance: $\mathrm{n} . \mathrm{s}$. - not significant $\mathrm{P}>0.05 ; * * * \mathrm{P}<0.001$.
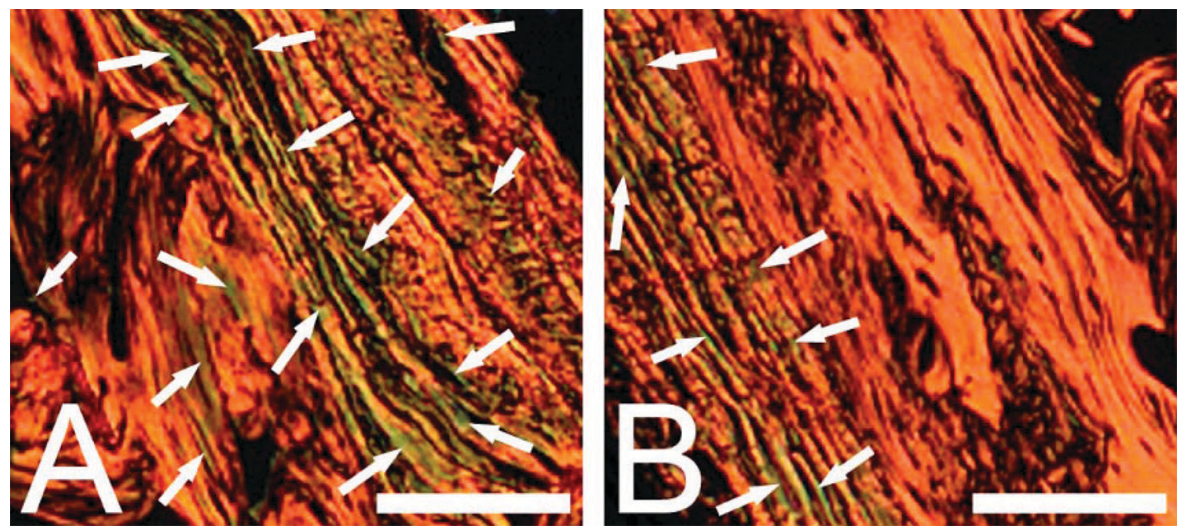

A - the control group, B - the FB group; scale bar - $20 \mu \mathrm{m}$.

Cancellous bone from control rats contained more immature (thin) collagen fibers (green color, white arrows). The content of immature collagen decreased in cancellous bone in FB-intoxicated rats. Thick collagen fibers stained in red or yellow.

Figure 3. Representative images of the Picrosirius red (PSR) staining carried out on formaldehydefixed sections from the cancellous bone of femur of adolescent rats FB-intoxicated for 21 days

Bone content of $\mathrm{Ca}, \mathrm{Cu}, \mathrm{Fe}, \mathrm{Mn}, \mathrm{Sr}$ and $\mathrm{Zn}$ was significantly decreased in FBintoxicated rats compared to the control (Table 8). The content of $\mathrm{Ba}, \mathrm{Cd}, \mathrm{Cr}, \mathrm{Mg}$, Mo, P, PB, S and Si was not affected by FB intoxication (Table 8). However, the ratio of $\mathrm{Ca} / \mathrm{P}$ was decreased.

The average hydroxyapatite crystallites size of the apatite-bone crystals, both in a-b plane and z axis, was also decreased in FB-intoxicated rats (Table 9). 
Table 8. Macro- and microelements in bone tissue obtained from adolescent control and FB-intoxicated rats

\begin{tabular}{l|c|c|c}
\hline \multirow{2}{*}{ Macro- and microelement } & \multicolumn{2}{c}{ Group } & \multirow{2}{*}{ P-value } \\
\cline { 2 - 3 } & control & fumonisin & n.s. \\
$\mathrm{na}(\mathrm{mg} / \mathrm{kg})$ & $11.3 \pm 0.4$ & $11.9 \pm 0.6$ & $* * *$ \\
$\mathrm{Ca}(\mathrm{g} / \mathrm{kg})$ & $315 \pm 3$ & $307 \pm 2$ & n.s. \\
$\mathrm{Cd}(\mathrm{mg} / \mathrm{kg})$ & $0.078 \pm 0.009$ & $0.061 \pm 0.009$ & n.s. \\
$\mathrm{Cr}(\mathrm{mg} / \mathrm{kg})$ & $6.91 \pm 0.19$ & $6.68 \pm 0.15$ & $* * *$ \\
$\mathrm{Cu}(\mathrm{mg} / \mathrm{kg})$ & $5.45 \pm 0.09$ & $1.82 \pm 0.08$ & $* * *$ \\
$\mathrm{Fe}(\mathrm{mg} / \mathrm{kg})$ & $52.6 \pm 0.3$ & $38.0 \pm 0.2$ & n.s. \\
$\mathrm{Mg}(\mathrm{g} / \mathrm{kg})$ & $5.37 \pm 0.3$ & $5.38 \pm 0.2$ & $* * *$ \\
$\mathrm{Mn}(\mathrm{mg} / \mathrm{kg})$ & $0.95 \pm 0.01$ & $0.81 \pm 0.01$ & n.s. \\
$\mathrm{Mo}(\mathrm{mg} / \mathrm{kg})$ & $0.34 \pm 0.04$ & $0.34 \pm 0.01$ & n.s. \\
$\mathrm{P}(\mathrm{g} / \mathrm{kg})$ & $142 \pm 3$ & $141 \pm 2$ & n.s. \\
$\mathrm{Pb}(\mathrm{mg} / \mathrm{kg})$ & $1.81 \pm 0.3$ & $2.03 \pm 0.5$ & n.s. \\
$\mathrm{S}(\mathrm{g} / \mathrm{kg})$ & $2.68 \pm 0.09$ & $2.70 \pm 0.03$ & n.s. \\
$\mathrm{Si}(\mathrm{mg} / \mathrm{kg})$ & $236 \pm 2$ & $238 \pm 2$ & $* * *$ \\
$\mathrm{Sr}(\mathrm{mg} / \mathrm{kg})$ & $158 \pm 2$ & $173 \pm 2$ & $* * *$ \\
$\mathrm{Zn}(\mathrm{mg} / \mathrm{kg})$ & $311 \pm 1$ & $295 \pm 1$ & $* * *$ \\
$\mathrm{Ca} / \mathrm{P}$ & $2.22 \pm 0.04$ & $2.18 \pm 0.08$ & \\
\hline
\end{tabular}

Statistical significance: $\mathrm{n} . \mathrm{s} .-$ not significant $\mathrm{P}>0.05 ; * * * \mathrm{P}<0.001$.

Table 9. Hydroxyapatite nanocrystallites size in femur obtained from adolescent control and FB-intoxicated rats

\begin{tabular}{|c|c|c|c|}
\hline \multirow{2}{*}{ Nanocrystallites size } & \multicolumn{2}{|c|}{ Group } & \multirow{2}{*}{ P-value } \\
\hline & control & fumonisin & \\
\hline a-b plane $(\mathrm{nm})$ & $21.09 \pm 0.73$ & $15.33 \pm 0.41$ & $* * *$ \\
\hline $\mathrm{z}$ axis $(\mathrm{nm})$ & $25.29 \pm 2.01$ & $20.88 \pm 1.26$ & $* *$ \\
\hline
\end{tabular}

Statistical significance: $\mathrm{n} . \mathrm{s}$. - not significant $\mathrm{P}>0.05 ; * * \mathrm{P}<0.01 ; * * * \mathrm{P}<0.001$.

\section{Results of blood serum analyses}

The serum Ca concentration in control rats reached the value of $2.55 \pm 0.11$ $\mathrm{mmol} / \mathrm{L}$ and differed significantly from the value obtained in the FB-intoxicated group $(2.21 \pm 0.20 \mathrm{mmol} / \mathrm{L} ; \mathrm{P}<0.05)$. Also the serum $\mathrm{P}$ concentration in the FB group was significantly decreased compared to the control group $(\mathrm{P}<0.001)$, and amounted to $2.08 \pm 0.23 \mathrm{mmol} / \mathrm{L}$ and $3.22 \pm 0.56 \mathrm{mmol} / \mathrm{L}$, respectively.

\section{Discussion}

A diagnosis of fumonisin toxicosis is difficult and could be based only on clinical signs, histopathology examination, or the presence of fumonisins at toxic levels in the feed. The treatment of fumonisin toxicosis also is difficult due to the lack of specific treatment or antidote. The best way is to avoid fumonisin toxicosis. For this, both the European Commission in the EU and FDA in the USA have issued guidance levels for total fumonisins in human foods and animal feed (EC, 2006; 2016; FDA, 
2001). Despite this, many studies have shown the potential impact of FBs exposure in the incidence of toxicosis and the mechanisms involved in their toxic effects, but mainly concerning liver or kidney, less bone homeostasis. Despite the fact that feed toxicities have been identified as one of the main factors affecting bone homeostasis (Tomaszewska et al., 2017 a), to the best of our knowledge, only two studies on the effect of FBs intoxication on bone quality have been conducted previously (Wu et al., 1995; Lebepe-Mazur et al., 1995).

The first information that FB1 has influenced body mass was reported by Gelderblom et al. (1994), who fed rats FB contaminated feed $(250 \mathrm{mg} / \mathrm{kg})$ for 21 days. However, study performed on male or female rats has shown that among diets containing FB1 at the level of 1, 9, 27 or $81 \mathrm{mg} / \mathrm{kg}$ /day for 13 weeks no difference was observed between control and intoxicated animals in body mass after treatment with the dose of $27 \mathrm{mg} \mathrm{FB1} / \mathrm{kg} /$ day (Voss et al., 1995). Also the other study has shown that FB1 at the level of 50 or $100 \mathrm{mg} / \mathrm{kg}$ of feed for 21 days does not affect the body mass (Abel and Gelderblom, 1998).

More detailed discussion concerning findings observed in our FB-intoxicated rats with data from other studies is somewhat difficult because no studies on the effects of FB intoxication on bone metabolism are available in satisfactory number.

Yet, the dose of fumonisins used in the present study were sufficient to trigger the disturbance in mineral homeostasis resulting in altered bone metabolism and decreased mechanical endurance. Although, FB intoxication did not affect bone mass, but reduced the length and geometry of femur in our rats. This change was supported by the structure analysis of the growth plate cartilage, the total thickness of which was reduced by $50 \%$. It could suggest appreciable slowdown in the processes of the proliferation and the degree of bone mineralization, general growth and bone development finally.

These observations are consistent with in vitro study with chondrocytes performed by $\mathrm{Wu}$ et al. (1995), who proved that FB1 (at the concentration of $250 \mu \mathrm{M}$ ) has reduced the viability of chondrocytes from the tibia of chickens. The reduction in cell size and division rate has been described (Wu et al., 1995). However, contrary to our results, Wu et al. (1995) have not observed the change in mineral status in their animals after inclusion of FB1 in the diet (110 ppm). They supposed that the lack of the effect of $\mathrm{FB}$ on in vivo bone formation can result from insufficient exposure of chondrocytes in the growth plate to mycotoxin.

But on the other hand, it is as important as unexpected, FB intoxication improved almost all histomorphometrical parameters of trabecular bone in our rats (Figure 2). The femoral trabeculae increments found in our FB exposed rats could be interpreted as a bone compensatory mechanism for the loss of cortical bone, trying to adapt the bone structure to the mechanical loads (Ferretti et al., 2003). However, once again these results could indicate that FB intoxication disturbed bone metabolism mainly affecting the mineral balance. The FB intoxication resulted in decreased serum $\mathrm{Ca}$ and $\mathrm{P}$ concentrations and decreased bone mineral status, which was shown by both densitometry and by ICP-OES analysis.

Additionally, the performed three-point bending test showed that bones in the FB group were characterized with lowered mechanical endurance and more "rubber- 
like" nature. The bones from rats from the FB group were significantly less rigid and prone to plastic deformations, as indicated by decreased values of yield load and elastic energy. This was a result of changes in bone geometry, as bones from the FB group were characterized with reduced length and altered spatial distribution of cortical bone tissue as indicated by size of the internal diameters, mean relative wall thickness, and vertical cortical index, which indicates larger medullary cavity in bone. Furthermore, analysis of material properties revealed that while the bones from both groups were breaking under the same load, bones from the FB group experienced greater distortions, as shown by significantly greater values of ultimate strain, and were characterized by increased risk of fracture, as a consequence of decreased energy absorption capacity by the whole bone (work to fracture). Once again, lowered mechanical endurance resulted directly from alterations in spatial distribution of the cortical bone mass, as both yield and ultimate stress, which correct raw loads for bone geometry (Muszyński et al., 2017), were significantly lower in the FB group.

However, the bone tendency to deformation and fracture under the action of external forces may be also associated with other factors, such as the alterations in mineralization or the structure of the organic phase. Both quantitative (bone mineral content, ash percentage) and qualitative (mineral composition) indicators of bone mineralization were negatively affected by FBs.

It has been found that one of the effects of mycotoxin action is disturbance of mineral homeostasis, including calcium and phosphorus (Smith, 1981; Sergeev et al., 1990). In our study, $\mathrm{Ca}$ and $\mathrm{P}$ homeostasis was negatively affected by FB action, as shown by decreased serum $\mathrm{Ca}$ and $\mathrm{P}$ concentrations and lowered $\mathrm{Ca}$ bone content. Furthermore, ICP-OES analysis revealed the disturbance of homeostasis of other ions closely related to bone metabolism ( $\mathrm{Cu}, \mathrm{Fe}, \mathrm{Mn}$, and $\mathrm{Zn}$ ) in FB groups. The only microelement whose content increased in FB group was $\mathrm{Sr}$, however, while it has been shown to replace some of the $\mathrm{Ca}$ ions in bone, but this substitution does not enhance bone structural and material strength (Shahnazari et al., 2007).

The present study also showed a significant decrease in the articular cartilage thickness and proteoglycan content in FB-intoxicated rats (Figure 3). This effect might trigger the degradation of articular cartilage changing the distribution of the load through the joint with functional consequences and difficulties in movement, particularly with increasing age. Additionally, the degradation of proteoglycans can play a pivotal role in destabilization of the collagen network, because they give stability of articular cartilage. Proteoglycans, whose function is to resist compressive forces in joint, provide hydration and swelling pressure to the tissue, making it more elastic. Together with collagen matrix proteoglycans help to stabilize and maintain the shape of the cartilage (Camplejohn and Allard, 1988; Yanagishita, 1993). Decreased proteoglycans content and the increase of mature collagen could predispose articular cartilage to the degradation. This effect is described for the first time. To our knowledge there is no available data concerning articular cartilage in FB toxicosis. Moreover, the thickness of the superficial zone (zone I), which plays an important role in lubrication and frictional characteristics of articular cartilage (Naka et al., 
2005), was also significantly lower in FB-intoxicated rats, which suggests that the hydration of the cartilage surface, might have been reduced in that group.

Bone inorganic phase is the dominant factor determining the bone fracture endurance while the bone elastic behavior is also dependent on bone organic phase, mostly collagen matrix (Hayes and Gerhart, 1985). It is proved that the connective tissue strength depends on the orientation, density, and length of both collagen fibrils and fibers (Jonas et al., 1993; Kjaer 2004). Furthermore, the content of thin collagen fibers provided additional structural information about the influence of FB intoxication on collagen synthesis (Table 7). The greater abundance of thick collagen fibers (red color) in the bone might indicate higher maturity of synthesized collagen occurring in FB-intoxicated rats (Figure 2). The occurrence of immature (green color) collagen in the bone observed in the control group could indicate that the bone turnover process is more intensive and the growth has not finished, as it was evidenced by longer bones.

We also aimed to examine whether the FBs intoxication could alter the hydroxyapatite structure in bones. The change in hydroxyapatite crystal domain size influences bone mechanical properties (Ruppel et al., 2008). As shown by XRD analyses (Table 9), differences in hydroxyapatite crystallites size were observed in our study, and smaller crystallites were found in the FB group. Generally, larger crystal will tend to reduce bone stiffness while too small crystals do not reinforce the bone composite material (Bhardwaj et al., 2016). This was observed in our study, as while the FB group was characterized by decreased mechanical endurance, stiffness was one of few determined mechanical parameters that were the same in both groups. The differences in hydroxyapatite crystallites size could be an effect of ionic incorporation into bone crystalline phase, as a number of bivalent cations, like Fe, $\mathrm{Mn}, \mathrm{Mg}$, and $\mathrm{Sr}$, can replace calcium ions in hydroxyapatite crystallite structures. However, it can be only speculated, as most of the elements can be located in both bone mineral and organic phases, which makes it impossible to assign them to specific component of bone.

Concluding, this is the first report showing that FB intoxication in rats, despite the improvement of trabecular histomorphometry, leads to demineralization and negative changes in femur mid-diaphysis geometry, which results in a decrease in bone mechanical endurance. Moreover, our study showed that FBs might affect the cells proliferation in growth plate and the synthesis of proteoglycans in articular cartilage.

\section{Conflict of interests statement}

The authors declare that they have no conflict of interests regarding the publication of this article.

\section{References}

A bel S., Ge ld e r blo m W.C. (1998). Oxidative damage and fumonisin B1-induced toxicity in primary rat hepatocytes and rat liver in vivo. Toxicology, 131: 121-131. 
Álvarez-Lloret P., Lind P.M., Nyberg I., Orberg J., Rodríguez-Navarro A.B. (2009). Effects of 3,3',4,4',5-pentachlorobiphenyl (PCB126) on vertebral bone mineralization and on thyroxin and vitamin D levels in Sprague-Dawley rats. Toxicol. Lett., 187: 63-68.

Antonissen G., Croubels S., Pasmans F., Ducatelle R., Eeckhaut V., Devreese M., Verlinden M., Haes ebrouck F., Eeckhout M., De Saeger S., Antlinger B., Novak B., Martel A., Van Immerse el F. (2015). Fumonisins affect the intestinal microbial homeostasis in broiler chickens, predisposing to necrotic enteritis. Vet. Res., 46: 98.

B hardw aj P., R a i D., G a rg M.L. (2016). Zinc improves the bone mechanical strength in ovariectomized rat model by restoring bone composition and hydroxyapatite crystallite dimension. Vitam. Miner., 5: 137.

Blicharski T., Tomaszewska E., Dobrowolski P., Hułas-Stasiak M., Muszyńs ki S. (2017). A metabolite of leucine ( $\beta$-hydroxy- $\beta$-methylbutyrate) given to sows during pregnancy alters bone development of their newborn offspring by hormonal modulation. PLOS ONE, 12: e0179693.

C a m p lejohn K.L., A 1 la rd S.A. (1988). Limitations of safranin 'O' staining in proteoglycan-depleted cartilage demonstrated with monoclonal antibodies. Histochemistry, 89: 185-188.

EC (European Commission) (2006). Commission Recommendation No. 576/2006 of 17 August 2006 on the presence of deoxynivalenol, zearalenone, ochratoxin A, T-2 and HT-2 and fumonisins in products intended for animal feeding. Off. J. Eur. Union, L229: 7.

EC (European Commission) (2016). Commission Recommendation No 2016/1219 of 29 July 2016 amending Recommendation 2006/576/EC as regards deoxynivalenol, zearalenone and ochratoxin A in pet food. Off. J. Eur. Union, L208: 59.

FDA (Food and Drug Administration) (2001). Guidance for industry: fumonisin levels in human foods and animal feeds. Federal Register, 66: 56688-56689.

F erretti J.L., Cointry G.R., Capozza R.F., Frost H.M. (2003). Bone mass, bone strength, muscle-bone interactions, osteopenias and osteoporoses. Mech. Ageing Dev., 124: 269-279.

G e ld e r b 1 o m W.C., Caw o od M.E., S n y m a n S.D., M a r a s s W.F. (1994). Fumonisin B1 dosimetry in relation to cancer initiation in rat liver. Carcinogenesis, 15: 209-214.

H a r kn e s s J.E., Wa g n e r J.E. (1995). The biology and medicine of rabbits and rodents. York, Williams and Wilkins, 4th ed., 372 pp.

Hayes W.C., Gerhart T.N. (1985). Biomechanics of bone: Applications for assessment of bone strength. J. Bone Miner. Res., 3: 259-294.

Hodgs on S., Thom a s L., F a t ore E., L ind P.M., A lfven T., Hells trö m L., Håk a n s s on H., Carubelli G., F a n elli R., J a ru p L. (2008). Bone mineral density changes in relation to environmental PCB exposure. Environ. Health Persp., 116: 1162-1166.

Jonas J., Burns J., Abel E.W., Cresswell M.J., Strain J.J., Paterson C.R. (1993). Impaired mechanical strength of bone in experimental copper deficiency. Ann. Nutr. Metab., 37 : $245-252$.

Ki erończyk B., Rawski M., Józefiak D., Świątkiewicz S. (2017). Infectious and non-infectious factors associated with leg disorders in poultry - a review. Ann. Anim. Sci., 17: $645-669$.

K j a e r M. (2004). Role of extracellular matrix in adaptation of tendon and skeletal muscle to mechanical loading. Physiol. Rev., 84: 649-698.

Lebepe-Mazur S., Bal H., Hopmans E., Murphy P., Hendrich S. (1995). Fumonisin B1 is fetotoxic in rats. Vet. Hum. Toxicol., 37: 126-130.

L e d o ux D.R., B rown T.P., We ibking T.S., R o t $\mathrm{t}$ ing ha u s G.E. (1992). Fumonisin toxicity in broiler chicks. J. Vet. Diagn. Invest., 4: 330-333.

Lee S., Kim D.H., Keum M.C., Han E., An B.K., Chang H.H., Choi Y.H., Moon B.H., L e e K.W. (2018). Effects of fumonisin B1 and mycotoxin binders on growth performance, tibia characteristics, gut physiology, and stress indicators in broiler chickens raised in different stocking densities. Poultry Sci., 97: 845-854.

McKean C., Tang L., Tang M., Billam M., Wang Z., Theodorakis C.W., Kend a 11 R.J., Wang J.S. (2006). Comparative acute and combinative toxicity of aflatoxin B1 and fumonisin B1 in animals and human cells. Food Chem. Toxicol., 44: 868-876.

Muszyński S., Kwiecień M., Tomaszewska E., Świetlicka I., Dobrowolski P., 
Kas perek K., J eżewska-Witk owska G. (2017). Effect of caponization on performance and quality characteristics of long bones in Polbar chickens. Poultry Sci., 96: 491-500.

$\mathrm{N}$ a k a M., M or it a Y., I k e u ch i K. (2005). Influence of proteoglycan contents and of tissue hydration on the frictional characteristics of articular cartilage. Proc. Inst. Mech. Eng. H., 2019: 175-182.

P 1 a c in t a C.M., D ' Me 11 o J.P.F., M a c d o n a ld A.M.C. (1999). A review of worldwide contamination of cereal grains and animal feed with Fusarium mycotoxins. Anim. Feed Sci. Technol., 78: 21-37.

Rajesh R., Hariharasubramanian A., Ravichandran Y.D. (2012). Chicken bone as a bioresource for the bioceramic (hydroxyapatite). Phosphorus Sulfur, 187: 914-925.

R e e v e s P.G., N i e 1 s e n F.H., F a h e y Jr. G.C. (1993). AIN-93 Purified diets for laboratory rodents: final report of the American Institute of Nutrition ad hoc writing committee on the reformulation of the AIN-76A rodent diet. J. Nutr., 123: 1939-1951.

R i ch L., Wih it t a k e r P. (2005). Collagen and picrosirius red staining: a polarized light assessment of fibrillar hue and spatial distribution. Braz. J. Morphol. Sci., 22: 97-104.

R i l e y R.T., Vo s s K.A. (2006). Differential sensitivity of rat kidney and liver to fumonisin toxicity: organ-specific differences in toxin accumulation and sphingoid base metabolism. Toxicol. Sci., 92: $335-345$.

Rodríguez-Estival J., Álvarez-Lloret P., Rodríguez-Navarro A.B., Mateo R. (2013). Chronic effects of lead $(\mathrm{Pb})$ on bone properties in red deer and wild boar: relationship with vitamins A and $\mathrm{D}_{3}$. Environ. Pollut., 174: 142-149.

Ros s P.F., Rice L.G., O swe iler G.D., Nel s on P.E. (1992). A review and update of animal toxicoses associated with fumonisin contaminated feeds and production of fumonisins by Fusarium isolates. Mycopathologia, 117: 109-114.

R u p p e 1 M.E., M ille r L.M., B u r r D.B. (2008). The effect of the microscopic and nanoscale structure on bone fragility. Osteoporos. Int., 19: 1251-1265.

$\mathrm{S} \mathrm{c} h \mathrm{n}$ e i d e r C.A., R a s b a n d W.S., E 1 i c e i r i K.W. (2012). NIH Image to ImageJ: 25 years of image analysis. Nat. Methods, 9: 671-675.

$\mathrm{S}$ chre in er W.N., J en kins R. (1983). Profile fitting for quantitative analysis in X-ray powder diffraction. Adv. X-Ray Analysis, 26: 141-147.

Š egvić M., P e peljnjak S. (2001). Fumonisins and their effects on animal health a brief review. Vet. Arhiv., 71: 299-323.

Sergeev I.N., Kravchenko L.V., Piliia N.M., Batukhanov A.B., Sobolev V.S., Kuz'mina E.E., I a kush ina L.M., S p irichev V.B., Tutel'i an V.A. (1990). The effect of the trichothecene mycotoxin deoxynivalenol (vomitoxin) on calcium homeostasis, vitamin D metabolism and receptors in rats. Vopr. Med. Khim., 36: 26-29.

Shahnazari M., Lang D., Fosmire G., Sharkey N., Mitchell A., Leach R. (2007). Strontium administration in young chickens improves bone volume and architecture but does not enhance bone structural and material strength. Calcif. Tissue Int., 80: 160-166.

$\mathrm{S} \mathrm{m}$ it th T.K. (1981). Effect of dietary calcium, phosphorus and vitamin D on zearalenone toxicosis in rats. Can. J. Anim. Sci., 61: 191-197.

Stre it E., N a ehre r K., R odrig u es I., S ch a t z may r G. (2013). Mycotoxin occurrence in feed and feed raw materials worldwide: Long-term analysis with special focus on Europe and Asia. J. Sci. Food Agric., 93: 2892-2899.

$\mathrm{S}$ u var a S.K., L a y t o n C., B a n c r o ft J.D. (2013). Bancroft's theory and practice of histological techniques. Edinburgh, Churchill Livingstone, 7th ed., pp. 654.

Śliwa E., Dobrowolski P., Tatara M.R., Piersiak T., Siwicki A., Rokita E, Pie r z y n o w s k i S.G. (2009). Alpha-ketoglutarate protects the liver of piglets exposed during prenatal life to chronic excess of dexamethasone from metabolic and structural changes. J. Anim. Physiol. Anim. Nutr., 93: 192-202.

To maszew sk a E., Dobrowolski P., Wy dry ch J. (2012). Postnatal administration of 2-oxoglutaric acid improves articular and growth plate cartilages and bone tissue morphology in pigs prenatally treated with dexamethasone. J. Physiol. Pharmacol., 63: 547-554.

To mas zew ska E., D obrow olski P., P u zi o I. (2013). Morphological changes of the cartilage and bone in newborn piglets evoked by experimentally induced glucocorticoid excess during pregnancy. J. Anim. Physiol. Anim. Nutr., 97: 785-796. 
Tomaszewska E., Dobrowolski P., Kostro K., Jakubczak A., Taszkun I., Jaworska-Adamu J., Żmuda A., Rycerz K., Muszyński S. (2015). The effect of HMB and 2-Ox administered during pregnancy on bone properties in primiparous and multiparous minks (Neovison vison). Bull.Vet. Inst. Pulawy, 59: 563-568.

Tomaszewska E., Dobrowolski P., Winiarska-Mieczan A., Kwiecień M., Tomczyk A., Muszyński S., Radzki R. (2016). Alteration in bone geometric and mechanical properties, histomorphometrical parameters of trabecular bone, articular cartilage, and growth plate in adolescent rats after chronic co-exposure to cadmium and lead in the case of supplementation with green, black, red and white tea. Environ. Toxicol. Pharmacol., 46: 36-44.

Tomaszewska E., Dobrowolski P., Muszyński S., Kostro K., Taszkun I., Żmud a A., B lich arski T., H u ł a s-S t a si a k M. (2017 a). DON-induced changes in bone homeostasis in mink dams, J. Vet. Res., 61: 357-362.

Tomaszewska E., Muszyński S., Ognik K., Dobrowolski P., Kwiecień M., Juśkiewicz J., Chocyk D., Świetlicki M., Blicharski T., Gładyszewska B. (2017 b). Comparison of the effect of dietary copper nanoparticles with copper (II) salt on bone geometric and structural parameters as well as material characteristics in a rat model. J. Trace Elem. Med. Biol., 42: 103-110.

To maszewska E., Dobrowolski P., Kwiecień M., Winiarska-Mieczan A., Tomc zyk A., Muszyński S., Gła d y szew sk a B. (2017 c). Dose-dependent influence of dietary $\mathrm{Cu}$-glycine complex on bone and hyaline cartilage development in adolescent rats. Ann. Anim. Sci., 17: $1089-1105$.

Tomaszewska E., Muszyński S., Dobrowolski P., Winiarska-Mieczan A., Kwiecień M., Tomczyk-Warunek A., Ejtel M., Świetlicka I., Gładyszew$\mathrm{s} \mathrm{k}$ a B. (2018 a). White tea is more effective in preservation of bone loss in adult rats co-exposed to lead and cadmium compared to black, red or green tea. Ann. Anim. Sci., 18: 937-953.

Tomaszewska E., Dobrowolski P., Świetlicka I., Muszyński S., Kostro K., Jakubczak A., Taszkun I., Żmuda A., Rycerz K., Blicharski T., Jaworska- A d a m u J. (2018 b). Effects of maternal treatment with $\beta$-hydroxy- $\beta$-metylbutyrate and 2-oxoglutaric acid on femur development in offspring of minks of the standard dark brown type. J. Anim. Physiol. Anim. Nutr., 102: e299-e308.

Vos s K.A., Ch a m b e r l a in W.J., B a c on C.W., H erbert R.A., Walt er s D.D., N or re d W.P. (1995). Species chronic feeding study of the mycotoxin FB1 in B6C3F1 mice and Fischer 344 rats. Fundam. Appl. Toxicol., 24: 102-110.

W u W., L i G., L i u T. (1995). The effect of fumonisin B1 on isolated chondrocytes and on bone formation. Poultry Sci., 74: 1431-1436.

Yanagishita M. (1993). Function of proteoglycans in the extracellular matrix. Acta Pathol. Jpn., 43: 283-293.

Received: 10 IX 2018

Accepted: 14 I 2019 